\title{
Some Congruence Properties of a Restricted Bipartition Function $c_{N}(n)$
}

\author{
Nipen Saikia and Chayanika Boruah \\ Department of Mathematics, Rajiv Gandhi University, Rono Hills, Doimukh, Arunachal Pradesh 791112, India \\ Correspondence should be addressed to Nipen Saikia; nipennak@yahoo.com
}

Received 12 April 2016; Accepted 10 July 2016

Academic Editor: Ahmed Zayed

Copyright (C) 2016 N. Saikia and C. Boruah. This is an open access article distributed under the Creative Commons Attribution License, which permits unrestricted use, distribution, and reproduction in any medium, provided the original work is properly cited.

Let $c_{N}(n)$ denote the number of bipartitions $(\lambda, \mu)$ of a positive integer $n$ subject to the restriction that each part of $\mu$ is divisible by $N$. In this paper, we prove some congruence properties of the function $c_{N}(n)$ for $N=7,11$, and $5 \ell$, for any integer $\ell \geq 1$, by employing Ramanujan's theta-function identities.

\section{Introduction}

A bipartition of a positive integer $n$ is an ordered pair of partitions $(\lambda, \mu)$ such that the sum of all of the parts equals $n$. If $c_{N}(n)$ counts the number of bipartitions $(\lambda, \mu)$ of $n$ subject to the restriction that each part of $\mu$ is divisible by $N$, then the generating function of $c_{N}(n)$ [1] is given by

$$
\sum_{n=0}^{\infty} c_{N}(n) q^{n}=\frac{1}{(q ; q)_{\infty}\left(q^{N} ; q^{N}\right)_{\infty}}
$$

where

$$
(a ; q)_{\infty}=\prod_{n=0}^{\infty}\left(1-a q^{n}\right)
$$

The partition function $c_{N}(n)$ is first studied by Chan [2] for the particular case $N=2$ by considering the function $c_{2}(n)$ defined by

$$
\sum_{n=0}^{\infty} c_{2}(n) q^{n}=\frac{1}{(q ; q)_{\infty}\left(q^{2} ; q^{2}\right)_{\infty}}
$$

Chan [2] proved that, for $n \geq 0$,

$$
c_{2}(3 n+2) \equiv 0(\bmod 3) .
$$

Kim [3] gave a combinatorial interpretation (4). In a subsequent paper, Chan [4] showed that, for $k \geq 1$ and $n \geq 0$,

$$
c_{2}\left(3^{k} n+s_{k}\right) \equiv 0\left(\bmod 3^{k+\delta(k)}\right),
$$

where $s_{k}$ is the reciprocal modulo $3^{k}$ of 8 and $\delta(k)=1$ if $k$ is even and 0 otherwise. Inspired by the work of Ramanujan on the standard partition function $p(n)$, Chan [4] asked whether there are any other congruence properties of the following form: $c_{2}(\ell n+k) \equiv 0(\bmod \ell)$, where $\ell$ is prime and $0 \leq$ $k \leq \ell$. Sinick [1] answered Chan's question in negative by considering restricted bipartition function $c_{N}(n)$ defined in (1). Liu and Wang [5] established several infinite families of congruence properties for $c_{5}(n)$ modulo 3 . For example, they proved that

$$
c_{5}\left(3^{2 \alpha+1} n+\frac{7 \cdot 3^{2 \alpha}+1}{4}\right) \equiv 0(\bmod 3),
$$

$$
\alpha \geq 1, n \geq 0 .
$$

Baruah and Ojah [6] also proved some congruence properties for some particular cases of $c_{N}(n)$ by considering the generalised partition function $p_{\left[c^{l} d^{m}\right]}(n)$ defined by

$$
\sum_{n=0}^{\infty} p_{\left[c^{l} d^{m}\right]}(n) q^{n}=\frac{1}{\left(q^{c} ; q^{c}\right)_{\infty}^{l}\left(q^{d} ; q^{d}\right)_{\infty}^{m}}
$$


and using Ramanujan's modular equations. Clearly, $c_{N}(n)=$ $p_{\left[1^{1} N^{1}\right]}(n)$. For example, Baruah and Ojah [6] proved that

$$
\begin{aligned}
& p_{\left[1^{1} 3^{1}\right]}(4 n+j) \equiv 0(\bmod 2), \quad \text { for } j=2,3, \\
& p_{\left[1^{1} 7^{1}\right]}(8 n+7) \equiv 0(\bmod 2) .
\end{aligned}
$$

Ahmed et al. [7] investigated the function $C_{N}(n)$ for $N=$ 3 and 4 and proved some congruence properties modulo 5 . They also gave alternate proof of some congruence properties due to Chan [2].

In this paper, we investigate the restricted bipartition function $c_{N}(n)$ for $n=7,11$, and $5 \ell$, for any integer $\ell \geq 1$, and prove some congruence properties modulo 2,3 , and 5 by using Ramanujan's theta-function identities. In Section 3, we prove congruence properties modulo 2 for $c_{7}(n)$. For example, we prove, for $\alpha \geq 0$,

$$
c_{7}\left(2^{2 \alpha+1} n+\frac{5 \cdot 2^{2 \alpha}+1}{3}\right) \equiv 0(\bmod 2) .
$$

In Section 4, we deal with the function $c_{11}(n)$ and establish the notion that if $p$ is an odd prime, $1 \leq j \leq p-1$, and $\alpha \geq 0$, then

$$
c_{11}\left(4 p^{2 \alpha+1}(p n+j)+\frac{p^{2 \alpha+2}+1}{2}\right) \equiv 0(\bmod 2) .
$$

In Section 5 , we show that, for any integer $\ell \geq 1, c_{5 l}(5 n+4) \equiv$ 0 (mod5). We also prove congruence properties modulo 3 for $c_{15}(n)$. Section 2 is devoted to listing some preliminary results.

\section{Preliminary Results}

Ramanujan's general theta function $f(a, b)$ is defined by

$$
f(a, b)=\sum_{n=0}^{\infty} a^{n(n+1) / 2} b^{n(n-1) / 2}, \quad|a b|<1 .
$$

Three important special cases of $f(a, b)$ are

$$
\begin{aligned}
\phi(q) & :=f(q, q)=\sum_{n=-\infty}^{\infty} q^{n^{2}}=\frac{\left(q^{2} ; q^{2}\right)_{\infty}^{5}}{(q ; q)_{\infty}^{2}\left(q^{4} ; q^{4}\right)_{\infty}^{2}}, \\
\psi(q) & :=f\left(q, q^{3}\right)=\sum_{n=0}^{\infty} q^{n(n+1) / 2}=\frac{\left(q^{2} ; q^{2}\right)_{\infty}^{2}}{(q ; q)_{\infty}}, \\
f(-q) & :=f\left(-q,-q^{2}\right)=\sum_{n=-\infty}^{\infty}(-1)^{n} q^{n(3 n+1) / 2} \\
& =(q ; q)_{\infty} .
\end{aligned}
$$

Ramanujan also defined the function $\chi(q)$ as

$$
\chi(q)=\left(-q ; q^{2}\right)_{\infty} .
$$

Lemma 1. For any prime $p$ and positive integer $m$, one has

$$
\left(q^{p m} ; q^{p m}\right)_{\infty} \equiv\left(q^{m} ; q^{m}\right)_{\infty}^{p}(\bmod p) .
$$

Proof. It follows easily from the binomial theorem.

Lemma 2 (see [8, page 315]). One has

$$
\begin{aligned}
\psi(q) \psi\left(q^{7}\right)= & \phi\left(q^{28}\right) \psi\left(q^{8}\right)+q \psi\left(q^{14}\right) \psi\left(q^{2}\right) \\
& +q^{6} \psi\left(q^{56}\right) \phi\left(q^{4}\right) .
\end{aligned}
$$

Lemma 3. One has

$$
\psi(q) \psi\left(q^{7}\right) \equiv(q ; q)_{\infty}^{3}\left(q^{7} ; q^{7}\right)_{\infty}^{3}(\bmod 2) .
$$

Proof. From (13), we have

$$
\psi(q) \psi\left(q^{7}\right)=\frac{\left(q^{2} ; q^{2}\right)_{\infty}^{2}\left(q^{14} ; q^{14}\right)_{\infty}^{2}}{(q ; q)_{\infty}\left(q^{7} ; q^{7}\right)_{\infty}} .
$$

Simplifying (19) using Lemma 1 with $p=2$, we arrive at the desired result.

Lemma 4 (see [9, page 286, Equation (60)]). One has

$$
\begin{aligned}
\phi(-q) & =\frac{(q ; q)_{\infty}^{2}}{\left(q^{2} ; q^{2}\right)_{\infty}}, \\
\psi(-q) & =\frac{(q ; q)_{\infty}\left(q^{4} ; q^{4}\right)_{\infty}}{\left(q^{2} ; q^{2}\right)_{\infty}}, \\
f(q) & =\frac{\left(q^{2} ; q^{2}\right)_{\infty}^{3}}{(q ; q)_{\infty}\left(q^{4} ; q^{4}\right)_{\infty}}, \\
\chi(q) & =\frac{\left(q^{2} ; q^{2}\right)_{\infty}^{2}}{(q ; q)_{\infty}\left(q^{4} ; q^{4}\right)_{\infty}} .
\end{aligned}
$$

Lemma 5 (see [10, page 372]). One has

$$
\begin{aligned}
\psi(q) \psi\left(q^{11}\right)= & \phi\left(q^{66}\right) \psi\left(q^{12}\right) \\
& +q f\left(q^{44}, q^{88}\right) f\left(q^{2}, q^{10}\right) \\
& +q^{22} f\left(q^{22}, q^{110}\right) f\left(q^{8}, q^{4}\right) \\
& +q^{15} \psi\left(q^{132}\right) \phi\left(q^{6}\right) .
\end{aligned}
$$

Lemma 6 (see [8, page 350, Equation (13)]). One has

$$
f\left(q, q^{2}\right)=\frac{\phi\left(-q^{3}\right)}{\chi(-q)},
$$

where

$$
\chi(-q)=\frac{(q ; q)_{\infty}}{\left(q^{2} ; q^{2}\right)_{\infty}} .
$$

Lemma 7. One has

$$
f\left(q^{11} ; q^{22}\right) \equiv\left(q^{11} ; q^{11}\right)_{\infty}(\bmod 2) .
$$


Proof. Employing (20) in Lemma 6 and performing simplification using Lemma 1 with $p=2$, we obtain

$$
f\left(q ; q^{2}\right) \equiv(q ; q)_{\infty}(\bmod 2) .
$$

Replacing $q$ by $q^{11}$ in (28), we arrive at the desired result.

Lemma 8 (see [8, page 51, Example (v)]). One has

$$
f\left(q, q^{5}\right)=\psi\left(-q^{3}\right) \chi(q) .
$$

Lemma 9. One has

$$
f\left(q, q^{5}\right) \equiv \frac{\left(q^{3} ; q^{3}\right)_{\infty}^{3}}{(q ; q)_{\infty}}(\bmod 2) .
$$

Proof. Employing (21) and (23) in Lemma 8, we obtain

$$
f\left(q, q^{5}\right)=\frac{\left(q^{3} ; q^{3}\right)_{\infty}\left(q^{12} ; q^{12}\right)_{\infty}\left(q^{2} ; q^{2}\right)_{\infty}^{2}}{\left(q^{6} ; q^{6}\right)_{\infty}(q ; q)_{\infty}\left(q^{4} ; q^{4}\right)_{\infty}} .
$$

Simplifying (31) using Lemma 1 with $p=2$, we complete the proof.

Lemma 10 (see [11, page 5, Equation (15)]). One has

$$
\frac{\left(q^{3} ; q^{3}\right)_{\infty}^{3}}{(q ; q)_{\infty}}=\frac{\left(q^{4} ; q^{4}\right)_{\infty}^{3}\left(q^{6} ; q^{6}\right)_{\infty}^{2}}{\left(q^{2} ; q^{2}\right)_{\infty}^{2}\left(q^{12} ; q^{12}\right)_{\infty}}+q \frac{\left(q^{12} ; q^{12}\right)_{\infty}^{3}}{\left(q^{4} ; q^{4}\right)_{\infty}} .
$$

Lemma 11 (see [12, Theorem 2.1]). For any odd prime $p$,

$$
\psi(q)
$$

$$
\begin{aligned}
= & \sum_{k=0}^{(p-3) / 2} q^{\left(k^{2}+k\right) / 2} f\left(q^{\left(p^{2}+(2 k+1) p\right) / 2}, q^{\left(p^{2}-(2 k+1) p\right) / 2}\right) \\
& +q^{\left(p^{2}-1\right) / 8} \psi\left(q^{p^{2}}\right),
\end{aligned}
$$

where, for $0 \leq k \leq(p-3) / 2$,

$$
\frac{k^{2}+k}{2} \neq \frac{p^{2}-1}{8}(\bmod p) .
$$

Lemma 12 (see [12, Theorem 2.2]). For any prime $p \geq 5$, one has

$$
\begin{aligned}
& f(-q)=\sum_{\substack{k=(-p-1) / 2 \\
k \neq( \pm p-1) / 6}}^{(p-1) / 2}(-1)^{k} \\
& \cdot q^{\left(3 k^{2}+k\right) / 2} f\left(-q^{\left(3 p^{2}+(6 k+1) p\right) / 2},-q^{\left(3 p^{2}-(6 k+1) p\right) / 2}\right) \\
& +(-1)^{( \pm p-1) / 6} q^{\left(p^{2}-1\right) / 24} f\left(-q^{p^{2}}\right),
\end{aligned}
$$

where

$$
\frac{ \pm p-1}{6}:= \begin{cases}\frac{p-1}{6}, & \text { if } p \equiv 1(\bmod 6) \\ \frac{-p-1}{6}, & \text { if } p \equiv-1(\bmod 6) .\end{cases}
$$

Lemma 13 (see [13]). One has

$$
\begin{aligned}
& \frac{1}{(q ; q)_{\infty}}=\frac{\left(q^{25} ; q^{25}\right)_{\infty}^{6}}{\left(q^{5} ; q^{5}\right)_{\infty}^{6}}\left(F^{4}\left(q^{5}\right)+q F^{3}\left(q^{5}\right)\right. \\
& +2 q^{2} F^{2}\left(q^{5}\right)+3 q^{3} F\left(q^{5}\right)+5 q^{4}-3 q^{5} F^{-1}\left(q^{5}\right) \\
& \left.+2 q^{6} F^{-2}\left(q^{5}\right)-q^{7} F^{-3}\left(q^{5}\right)+q^{8} F^{-4}\left(q^{5}\right)\right),
\end{aligned}
$$

where $F(q):=q^{-1 / 5} R(q)$ and $R(q)$ is Rogers-Ramanujan continued fraction defined by

$$
R(q):=\frac{q^{1 / 5}}{1+\frac{q}{1+\frac{q^{2}}{1+\frac{q^{3}}{1+\cdots}}}}, \quad|q|<1 .
$$

Lemma 14 (see [8, page 345, Entry 1(iv)]). One has

$$
(q ; q)_{\infty}^{3}=\left(q^{9} ; q^{9}\right)_{\infty}^{3}\left(4 q^{3} W^{2}\left(q^{3}\right)-3 q+W^{-1}\left(q^{3}\right)\right),
$$

where $W(q)=q^{-1 / 3} G(q)$ and $G(q)$ is Ramanujan's cubic continued fraction defined by

$$
G(q):=\frac{q^{1 / 3}}{1+\frac{q+q^{2}}{1+\frac{q^{2}+q^{4}}{1+\cdots}}}, \quad|q|<1 .
$$

\section{Congruence Identities for $c_{7}(n)$}

Theorem 15. One has

$$
\sum_{n=0}^{\infty} c_{7}(2 n+1) q^{n} \equiv(q ; q)_{\infty}\left(q^{7} ; q^{7}\right)_{\infty}(\bmod 2) .
$$

Proof. For $N=7$ in (1), we have

$$
\sum_{n=0}^{\infty} \mathcal{c}_{7}(n) q^{n}=\frac{1}{(q ; q)_{\infty}\left(q^{7} ; q^{7}\right)_{\infty}} .
$$

Employing (19) in (42), we obtain

$$
\sum_{n=0}^{\infty} c_{7}(n) q^{n}=\frac{\psi(q) \psi\left(q^{7}\right)}{\left(q^{2} ; q^{2}\right)_{\infty}^{2}\left(q^{14} ; q^{14}\right)_{\infty}^{2}} .
$$

Employing Lemma 2 in (43), we obtain

$$
\begin{aligned}
& \sum_{n=0}^{\infty} c_{\mathcal{7}}(n) q^{n}=\frac{1}{\left(q^{2} ; q^{2}\right)_{\infty}^{2}\left(q^{14} ; q^{14}\right)_{\infty}^{2}}\left[\phi\left(q^{28}\right) \psi\left(q^{8}\right)\right. \\
& \left.+q \psi\left(q^{14}\right) \psi\left(q^{2}\right)+q^{6} \psi\left(q^{56}\right) \phi\left(q^{4}\right)\right] .
\end{aligned}
$$

Extracting the terms involving $q^{2 n+1}$, dividing by $q$, and replacing $q^{2}$ by $q$ in (44), we get

$$
\sum_{n=0}^{\infty} \mathcal{G}_{7}(2 n+1) q^{n}=\frac{1}{(q ; q)_{\infty}^{2}\left(q^{7} ; q^{7}\right)_{\infty}^{2}}\left[\psi\left(q^{7}\right) \psi(q)\right] .
$$

Employing Lemma 3 in (45), we complete the proof. 
Theorem 16. One has

$$
\begin{aligned}
& \text { (i) } \sum_{n=0}^{\infty} c_{7}(4 n+3) q^{n} \\
& \equiv\left(q^{2} ; q^{2}\right)_{\infty}\left(q^{14} ; q^{14}\right)_{\infty}(\bmod 2), \\
& \text { (ii) } c_{7}(8 n+7) \equiv 0(\bmod 2) .
\end{aligned}
$$

Proof. From Theorem 15, we obtain

$$
\sum_{n=0}^{\infty} c_{7}(2 n+1) q^{n} \equiv \frac{\left(q^{7} ; q^{7}\right)_{\infty}^{3}(q ; q)_{\infty}^{3}}{\left(q^{7} ; q^{7}\right)_{\infty}^{2}(q ; q)_{\infty}^{2}}(\bmod 2) .
$$

Employing Lemma 3 in (47), we obtain

$$
\sum_{n=0}^{\infty} c_{7}(2 n+1) q^{n} \equiv \frac{\psi(q) \psi\left(q^{7}\right)}{\left(q^{2} ; q^{2}\right)_{\infty}\left(q^{14} ; q^{14}\right)_{\infty}}(\bmod 2) .
$$

Employing Lemma 2 in (48), extracting the terms involving $q^{2 n+1}$, dividing by $q$, and replacing $q^{2}$ by $q$, we obtain

$$
\begin{aligned}
& \sum_{n=0}^{\infty} \mathcal{c}_{7}(4 n+3) q^{n} \\
& \quad \equiv \frac{1}{(q ; q)_{\infty}\left(q^{7} ; q^{7}\right)_{\infty}} \psi(q) \psi\left(q^{7}\right)(\bmod 2) .
\end{aligned}
$$

Employing Lemma 3 in (49) and performing simplification using Lemma 1 with $p=2$, we arrive at (i).

All the terms on the right hand side of (i) are of the form $q^{2 n}$. Extracting the terms involving $q^{2 n+1}$ on both sides of (i), we complete the proof of (ii).

Theorem 17. For all $n \geq 0$, one has

(i) $c_{7}(14 n+7) \equiv 0(\bmod 2)$,

(ii) $c_{7}(14 n+9) \equiv 0(\bmod 2)$,

(iii) $c_{7}(14 n+13) \equiv 0(\bmod 2)$.

Proof. Employing (14) in Theorem 15, we obtain

$$
\begin{aligned}
& \sum_{n=0}^{\infty} \mathcal{c}_{7}(2 n+1) q^{n} \\
& \quad \equiv\left(q^{7} ; q^{7}\right)_{\infty} \sum_{n=0}^{\infty}(-1)^{n} q^{n(3 n+1) / 2}(\bmod 2) .
\end{aligned}
$$

Extracting those terms on each side of (50) whose power of $q$ is of the forms $7 n+3,7 n+4$, and $7 n+6$ and employing the fact that there exists no integer $n$ such that $n(3 n+1) / 2$ is congruent to 3,4 , and 6 modulo 7 , we obtain

$$
\begin{aligned}
\sum_{n=0}^{\infty} c_{7}(14 n+7) q^{7 n+3} & \equiv \sum_{n=0}^{\infty} c_{7}(14 n+9) q^{7 n+4} \\
& \equiv \sum_{n=0}^{\infty} c_{7}(14 n+13) q^{7 n+6} \\
& \equiv 0(\bmod 2) .
\end{aligned}
$$

Now, (i), (ii), and (iii) are obvious from (51).
Theorem 18. For $\alpha \geq 1$, one has

$$
\begin{aligned}
& \sum_{n=0}^{\infty} c_{7}\left(2^{2 \alpha+1} n+\frac{2^{2 \alpha+1}+1}{3}\right) q^{n} \\
& \equiv(q ; q)_{\infty}\left(q^{7} ; q^{7}\right)_{\infty}(\bmod 2) .
\end{aligned}
$$

Proof. We proceed by induction on $\alpha$. Extracting the terms involving $q^{2 n}$ and replacing $q^{2}$ by $q$ in Theorem 16(i), we obtain

$$
\sum_{n=0}^{\infty} c_{7}(8 n+3) q^{n} \equiv(q ; q)_{\infty}\left(q^{7} ; q^{7}\right)_{\infty}(\bmod 2),
$$

which corresponds to the case $\alpha=1$. Assume that the result is true for $\alpha=k \geq 1$, so that

$$
\begin{aligned}
& \sum_{n=0}^{\infty} c_{7}\left(2^{2 k+1} n+\frac{2^{2 k+1}+1}{3}\right) q^{n} \\
& \equiv(q ; q)_{\infty}\left(q^{7} ; q^{7}\right)_{\infty}(\bmod 2) .
\end{aligned}
$$

Employing Lemma 3 in (54), we obtain

$$
\begin{gathered}
\sum_{n=0}^{\infty} \mathcal{c}_{7}\left(2^{2 k+1} n+\frac{2^{2 k+1}+1}{3}\right) q^{n} \\
\equiv \frac{\psi(q) \psi\left(q^{7}\right)}{(q ; q)_{\infty}^{2}\left(q^{7} ; q^{7}\right)_{\infty}^{2}}(\bmod 2) .
\end{gathered}
$$

Employing Lemma 2 in (55) and extracting the terms involv$\operatorname{ing} q^{2 n+1}$, dividing by $q$, and replacing $q^{2}$ by $q$, we obtain

$$
\begin{gathered}
\sum_{n=0}^{\infty} c_{7}\left(2^{2 k+1}(2 n+1)+\frac{2^{2 k+1}+1}{3}\right) q^{n} \\
\equiv \frac{\psi(q) \psi\left(q^{7}\right)}{(q ; q)_{\infty}\left(q^{7} ; q^{7}\right)_{\infty}}(\bmod 2) .
\end{gathered}
$$

Simplifying (56) using Lemmas 3 and 1 with $p=2$, we obtain

$$
\begin{aligned}
& \sum_{n=0}^{\infty} c_{7}\left(2^{2(k+1)} n+\frac{2^{2(k+1)+1}+1}{3}\right) q^{n} \\
& \quad \equiv\left(q^{2} ; q^{2}\right)_{\infty}\left(q^{14} ; q^{14}\right)_{\infty}(\bmod 2) .
\end{aligned}
$$

Extracting the terms involving $q^{2 n}$ and replacing $q^{2}$ by $q$ in (57), we obtain

$$
\begin{gathered}
\sum_{n=0}^{\infty} c_{7}\left(2^{2(k+1)+1} n+\frac{2^{2(k+1)+1}+1}{3}\right) q^{n} \\
\equiv(q ; q)_{\infty}\left(q^{7} ; q^{7}\right)_{\infty}(\bmod 2),
\end{gathered}
$$

which is the $\alpha=k+1$ case. Hence, the proof is complete.

Theorem 19. For $\alpha \geq 0$, one has

$$
c_{7}\left(2^{2 \alpha+1} n+\frac{5 \cdot 2^{2 \alpha}+1}{3}\right) \equiv 0(\bmod 2) \text {. }
$$


Proof. All the terms in the right hand side of (57) are of the form $q^{2 n}$, so, extracting the coefficients of $q^{2 n+1}$ on both sides of (57) and replacing $k$ by $\alpha$, we obtain

$$
c_{7}\left(2^{2(\alpha+1)+1} n+\frac{5 \cdot 2^{2(\alpha+1)}+1}{3}\right) \equiv 0(\bmod 2) .
$$

Replacing $\alpha+1$ by $\alpha$ in (60) completes the proof.

Theorem 20. If any prime $p \geq 5,(-7 / p)=-1$, and $\alpha \geq 0$, then

$$
c_{7}\left(2^{2 \alpha+1} p^{2} n+\frac{2^{2 \alpha+1} p(3 j+p)+1}{3}\right) \equiv 0(\bmod 2),
$$

where $1 \leq j \leq p-1$.

Proof. Employing Lemma 12 in (52), we obtain

$$
\begin{aligned}
& \sum_{n=0}^{\infty} c_{7}\left(2^{2 \alpha+1} n+\frac{2^{2 \alpha+1}+1}{3}\right) q^{n} \equiv\left(\sum_{\substack{k=(-p-1) / 2 \\
k \neq( \pm p-1) / 6}}^{(p-1) / 2}(-1)^{k}\right. \\
& \cdot q^{\left(3 k^{2}+k\right) / 2} f\left(-q^{\left(3 p^{2}+(6 k+1) p\right) / 2},-q^{\left(3 p^{2}-(6 k+1) p\right) / 2}\right) \\
& \left.+(-1)^{( \pm p-1) / 6} q^{\left(p^{2}-1\right) / 24} f\left(-q^{p^{2}}\right)\right) \\
& \cdot\left(\sum_{\substack{k=(-p-1) / 2 \\
k \neq( \pm p-1) / 6}}^{(p-1) / 2}(-1)^{m}\right. \\
& \cdot q^{7 \cdot\left(3 m^{2}+m\right) / 2} f\left(-q^{7 \cdot\left(3 p^{2}+(6 m+1) p\right) / 2},\right. \\
& \left.-q^{7 \cdot\left(3 p^{2}-(6 m+1) p\right) / 2}\right)+(-1)^{( \pm p-1) / 6} \\
& \left.\cdot q^{7 \cdot\left(p^{2}-1\right) / 24} f\left(-q^{7 p^{2}}\right)\right)(\bmod 2) .
\end{aligned}
$$

We consider the congruence

$$
\frac{3 k^{2}+k}{2}+7 \cdot \frac{3 m^{2}+m}{2} \equiv \frac{8 p^{2}-8}{24}(\bmod p),
$$

where $-(p-1) / 2 \leq k, m \leq(p-1) / 2$. The congruence (63) is equivalent to

$$
(6 k+1)^{2}+7(6 m+1)^{2} \equiv 0(\bmod p)
$$

and, for $(-7 / p)=-1$, the congruence (64) has unique solution $k=m=( \pm p-1) / 6$. Extracting terms containing $q^{p n+\left(p^{2}-1\right) / 3}$ from both sides of (62) and replacing $q^{p}$ by $q$, we obtain

$$
\begin{gathered}
\sum_{n=0}^{\infty} c_{7}\left(2^{2 \alpha+1} p n+\frac{2^{2 \alpha+1} p^{2}+1}{3}\right) q^{n} \\
\equiv\left(q^{p} ; q^{p}\right)_{\infty}\left(q^{7 p} ; q^{7 p}\right)(\bmod 2) .
\end{gathered}
$$

Extracting the coefficients of $q^{p n+j}$, for $1 \leq j \leq p-1$, on both sides of (65) and performing simplification, we arrive at the desired result.

\section{Congruence Identities for $c_{11}(n)$}

Theorem 21. One has

$$
\sum_{n=0}^{\infty} c_{11}(4 n+1) q^{n} \equiv \frac{\left(q^{2} ; q^{2}\right)_{\infty}^{2}}{(q ; q)_{\infty}}=\psi(q)(\bmod 2) .
$$

Proof. Setting $N=11$ in (1), we obtain

$$
\sum_{n=0}^{\infty} c_{11}(n) q^{n}=\frac{1}{(q ; q)_{\infty}\left(q^{11} ; q^{11}\right)_{\infty}}
$$

Employing (13) in (67), we obtain

$$
\sum_{n=0}^{\infty} c_{11}(n) q^{n}=\frac{\psi(q) \psi\left(q^{11}\right)}{\left(q^{2} ; q^{2}\right)_{\infty}^{2}\left(q^{22} ; q^{22}\right)_{\infty}^{2}} .
$$

Employing Lemma 5 in (68), extracting the terms involving $q^{2 n+1}$, dividing by $q$, and replacing $q^{2}$ by $q$, we obtain

$$
\begin{aligned}
& \sum_{n=0}^{\infty} c_{11}(2 n+1) q^{n} \\
& \quad=\frac{1}{(q ; q)_{\infty}^{2}\left(q^{11} ; q^{11}\right)_{\infty}^{2}}\left[f\left(q^{22}, q^{44}\right) f\left(q, q^{5}\right)\right. \\
& \left.\quad+q^{7} \psi\left(q^{66}\right) \phi\left(q^{3}\right)\right] .
\end{aligned}
$$

Employing Lemmas 9 and 10 in (69), we find that

$$
\begin{aligned}
& \sum_{n=0}^{\infty} c_{11}(2 n+1) q^{n} \\
& \equiv \frac{1}{\left(q^{2} ; q^{2}\right)_{\infty}\left(q^{22} ; q^{22}\right)_{\infty}}\left[f\left(q^{22}, q^{44}\right)\right. \\
& \cdot\left(\frac{\left(q^{4} ; q^{4}\right)_{\infty}^{3}\left(q^{6} ; q^{6}\right)_{\infty}^{2}}{\left(q^{2} ; q^{2}\right)_{\infty}^{2}\left(q^{12} ; q^{12}\right)_{\infty}}+q \frac{\left(q^{12} ; q^{12}\right)^{3}}{\left(q^{4} ; q^{4}\right)_{\infty}}\right) \\
& \left.+q^{7} \psi\left(q^{66}\right) \phi\left(q^{3}\right)\right](\bmod 2) .
\end{aligned}
$$


Extracting the terms involving $q^{2 n}$ and replacing $q^{2}$ by $q$ on both sides of (70) and performing simplification using Lemma 1 with $p=2$, we obtain

$$
\begin{aligned}
\sum_{n=0}^{\infty} c_{11}(4 n+1) q^{n} \equiv & \frac{1}{(q ; q)_{\infty}\left(q^{11} ; q^{11}\right)_{\infty}} f\left(q^{11} ; q^{22}\right) \\
& \cdot\left(q^{2} ; q^{2}\right)_{\infty}^{2}(\bmod 2) .
\end{aligned}
$$

Employing Lemma 7 in (71) and using (13), we complete the proof.

Theorem 22. For any odd prime $p$ and any integer $\alpha \geq 0$, one has

$$
\sum_{n=0}^{\infty} c_{11}\left(4 p^{2 \alpha} n+\frac{p^{2 \alpha}+1}{2}\right) q^{n} \equiv \psi(q)(\bmod 2) .
$$

Proof. We proceed by induction on $\alpha$. The case $\alpha=0$ corresponds to the congruence theorem (Theorem 21). Suppose that the theorem holds for $\alpha=k \geq 0$, so that

$$
\sum_{n=0}^{\infty} c_{11}\left(4 p^{2 k} n+\frac{p^{2 k}+1}{2}\right) q^{n} \equiv \psi(q)(\bmod 2) .
$$

Employing Lemma 11 in (73), extracting the terms involving $q^{p n+\left(p^{2}-1\right) / 8}$ on both sides of (73), dividing by $q^{\left(p^{2}-1\right) / 8}$, and replacing $q^{p}$ by $q$, we obtain

$$
\sum_{n=0}^{\infty} c_{11}\left(4 p^{2 k+1} n+\frac{p^{2(k+1)}+1}{2}\right) q^{n} \equiv \psi\left(q^{p}\right)(\bmod 2) .
$$

Extracting the terms containing $q^{p n}$ from both sides of (74) and replacing $q^{p}$ by $q$, we arrive at

$$
\begin{aligned}
& \sum_{n=0}^{\infty} c_{11}\left(4 p^{2(k+1)} n+\frac{p^{2(k+1)}+1}{2}\right) q^{n} \\
& \equiv \psi(q)(\bmod 2),
\end{aligned}
$$

which shows that the theorem is true for $\alpha=k+1$. Hence, the proof is complete.

Theorem 23. For any odd prime $p$ and integers $\alpha \geq 0$ and $1 \leq j \leq p-1$, one has

$$
c_{11}\left(4 p^{2 \alpha+1}(p n+j)+\frac{p^{2 \alpha+2}+1}{2}\right) \equiv 0(\bmod 2) .
$$

Proof. Extracting the coefficients of $q^{p n+j}$ for $1 \leq j \leq p-1$ on both sides of (74) and replacing $k$ by $\alpha$, we arrive at the desired result.

\section{Congruence Identities for $c_{5 l}(n)$}

Theorem 24. For any positive integer $\ell$, one has

$$
c_{5 l}(5 n+4) \equiv 0(\bmod 5) \text {. }
$$

Proof. Setting $N=5 l$ in (1), we obtain

$$
\sum_{n=0}^{\infty} c_{5 l}(n) q^{n}=\frac{1}{(q ; q)_{\infty}\left(q^{5 l} ; q^{5 l}\right)_{\infty}} .
$$

Using Lemma 13 in (78) and extracting the terms involving $q^{5 n+4}$, dividing by $q^{4}$, and replacing $q^{5}$ by $q$, we obtain

$$
\sum_{n=0}^{\infty} c_{5 l}(5 n+4) q^{n}=5 \frac{\left(q^{5} ; q^{5}\right)_{\infty}^{6}}{\left(q^{l}, q^{l}\right)_{\infty}(q ; q)_{\infty}^{6}} .
$$

The desired result follows easily from (79).

Theorem 25. For all $n \geq 0$, one has

$$
\begin{aligned}
& \text { (i) } c_{15}(5 n+4) \equiv 0(\bmod 5), \\
& \text { (ii) } c_{15}(15 n+9) \equiv 0(\bmod 3), \\
& \text { (iii) } c_{15}(15 n+14) \equiv 0(\bmod 3) \text {. }
\end{aligned}
$$

Proof. Setting $N=15$ in (1), we obtain

$$
\sum_{n=0}^{\infty} c_{15}(n) q^{n}=\frac{1}{(q ; q)_{\infty}\left(q^{15} ; q^{15}\right)_{\infty}} .
$$

Employing Lemma 13 in (80), extracting terms involving $q^{5 n+4}$, dividing by $q^{4}$, and replacing $q^{5}$ by $q$, we obtain

$$
\sum_{n=0}^{\infty} c_{15}(5 n+4) q^{n}=5 \frac{\left(q^{5} ; q^{5}\right)_{\infty}^{6}}{\left(q^{3} ; q^{3}\right)_{\infty}(q ; q)_{\infty}^{6}} .
$$

Now, (i) follows from (81).

Simplifying (81) by using Lemma 1 with $p=3$, we obtain

$$
\begin{aligned}
\sum_{n=0}^{\infty} c_{15}(5 n+4) q^{n} & \equiv 2 \frac{\left(q^{15} ; q^{15}\right)_{\infty}^{2}}{(q ; q)_{\infty}^{3}(q ; q)_{\infty}^{6}} \frac{(q ; q)_{\infty}^{3}}{(q ; q)_{\infty}^{3}} \\
& =2 \frac{\left(q^{15} ; q^{15}\right)_{\infty}^{2}(q ; q)_{\infty}^{3}}{\left(q^{3} ; q^{3}\right)_{\infty}^{4}}(\bmod 3) .
\end{aligned}
$$

Employing Lemma 14 in (82) and performing simplification, we obtain

$$
\begin{aligned}
& \sum_{n=0}^{\infty} c_{15}(5 n+4) q^{n} \equiv 2 \\
& \cdot \frac{\left(q^{15} ; q^{15}\right)_{\infty}^{2}\left(q^{9} ; q^{9}\right)_{\infty}^{3}}{\left(q^{3} ; q^{3}\right)_{\infty}^{4}}\left[q^{3} W^{2}\left(q^{3}\right)\right. \\
& \left.+W^{-1}\left(q^{3}\right)\right](\bmod 3) .
\end{aligned}
$$

Extracting terms involving $q^{3 n+1}$ and $q^{3 n+2}$ on both sides of (83), we arrive at (ii) and (iii), respectively.

\section{Competing Interests}

The authors declare that there are no competing interests regarding the publication of this paper. 


\section{Acknowledgments}

The first author (Nipen Saikia) is thankful to the Council of Scientific and Industrial Research of India for partially supporting the research work under Research Scheme no. 25(0241)/15/EMR-II (F. no. 25(5498)/15).

\section{References}

[1] J. Sinick, "Ramanujan congruences for a class of eta quotients," International Journal of Number Theory, vol. 6, no. 4, pp. 835847, 2010.

[2] H.-C. Chan, "Ramanujan's cubic continued fraction and an analog of his 'most beautiful identity"' International Journal of Number Theory, vol. 6, no. 3, pp. 673-680, 2010.

[3] B. Kim, "A crank analog on a certain kind of partition function arising from the cubic continued fraction," Acta Arithmetica, vol. 148, pp. 1-19, 2011.

[4] H.-C. Chan, "Ramanujan's cubic continued fraction and Ramanujan type congruences for a certain partition function," International Journal of Number Theory, vol. 6, no. 4, pp. 819$834,2010$.

[5] J. Liu and A. Y. Z. Wang, "Arithmetic properties of a restricted bipartition function," The Electronic Journal of Combinatorics, vol. 22, no. 3, pp. 1-11, 2015.

[6] N. D. Baruah and K. K. Ojah, "Analogues of Ramanujan's partition identities and congruences arising from his theta functions and modular equations," Ramanujan Journal, vol. 28, no. 3, pp. 385-407, 2012.

[7] Z. Ahmed, N. D. Baruah, and M. G. Dastidar, "New congruences modulo 5 for the number of 2-color partitions," Journal of Number Theory, vol. 157, pp. 184-198, 2015.

[8] B. C. Berndt, Ramanujan's Notebooks, Part III, Springer, New York, NY, USA, 1991.

[9] N. D. Baruah, J. Bora, and N. Saikia, "Some new proofs of modular relations for the Göllnitz-Gordon functions," Ramanujan Journal, vol. 15, no. 2, pp. 281-301, 2008.

[10] B. C. Berndt, Ramanujan's Notebooks, Part V, Springer, New York, NY, USA, 1998.

[11] M. Hirschhorn, F. Garvan, and J. Borwein, "Cubic analogues of the Jacobian theta function $\theta(z ; q)$," Canadian Journal of Mathematics, vol. 45, no. 4, pp. 673-694, 1993.

[12] S.-P. Cui and N. S. S. Gu, "Arithmetic properties of $l$-regular partitions," Advances in Applied Mathematics, vol. 51, no. 4, pp. 507-523, 2013.

[13] M. D. Hirschhorn, "An identity of Ramanujan, and application in q-series from a contemporary perspective," Contemporary Mathematics, vol. 254, pp. 229-234, 2000. 


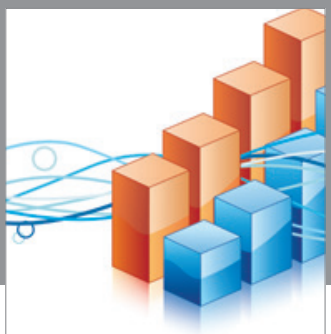

Advances in

Operations Research

vatem alat4

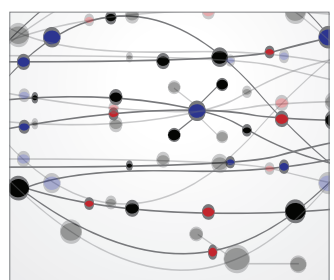

\section{The Scientific} World Journal
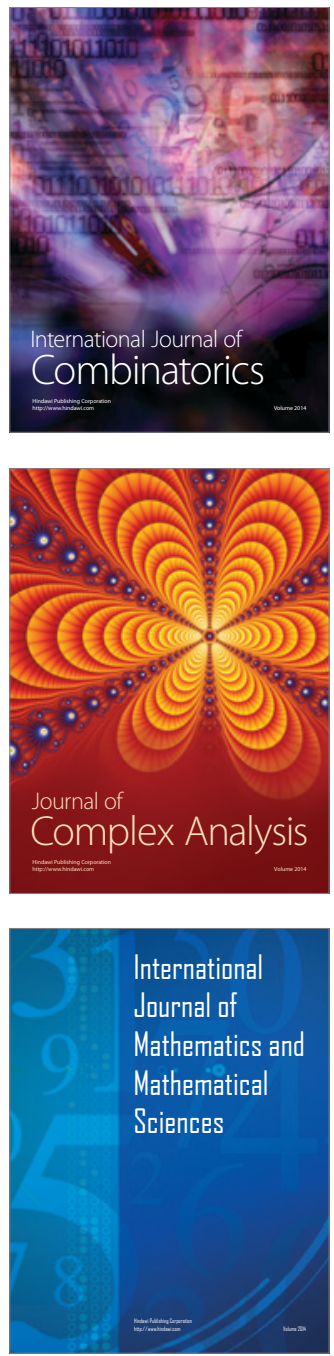
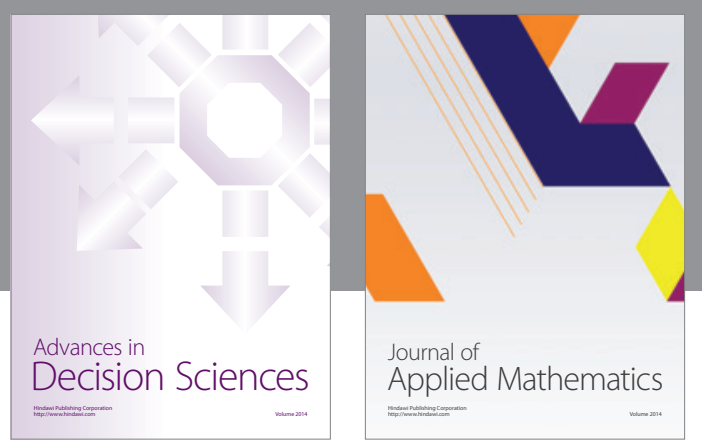

Algebra

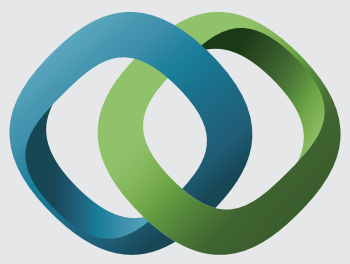

\section{Hindawi}

Submit your manuscripts at

http://www.hindawi.com
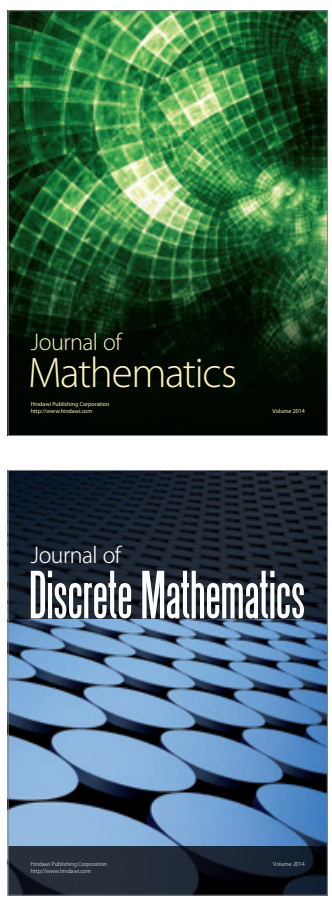

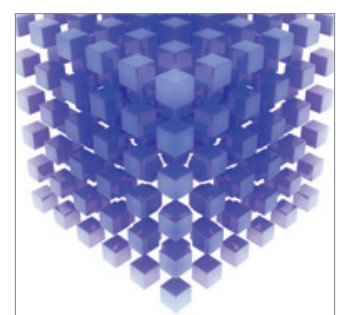

Mathematical Problems in Engineering
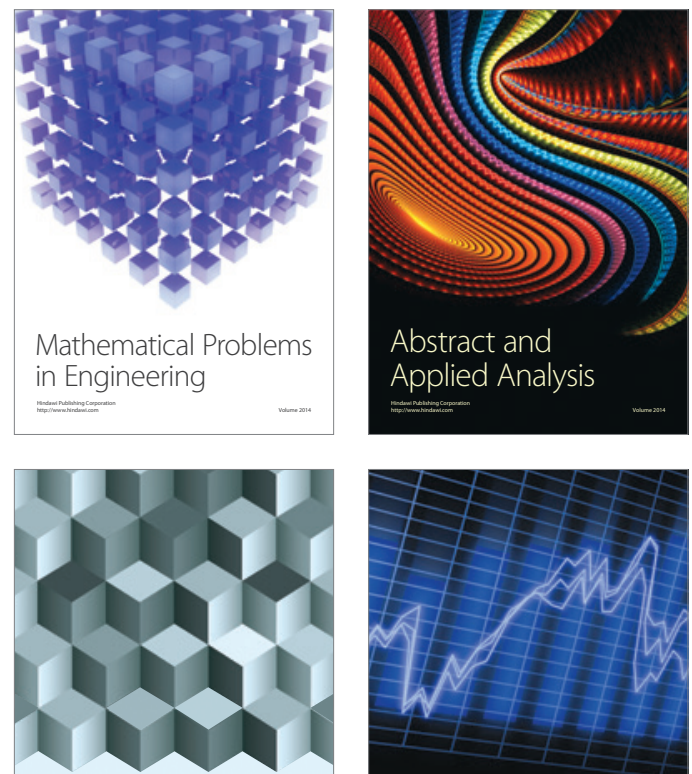

Journal of

Function Spaces

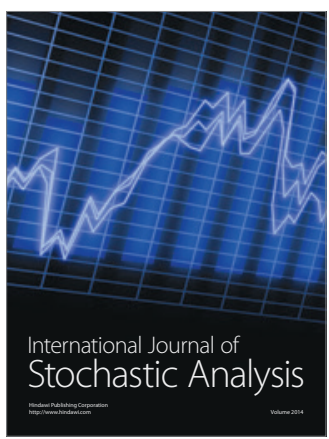

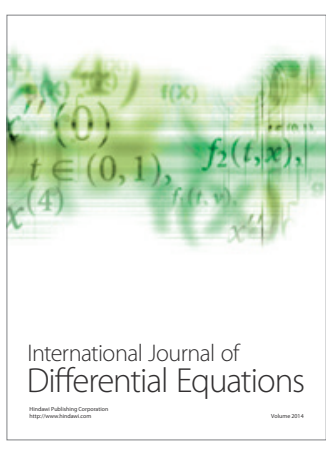
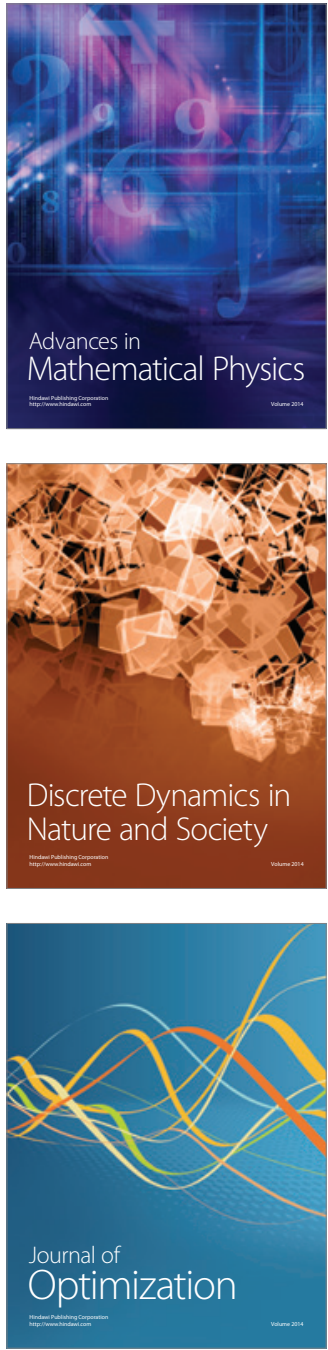